\title{
Quelle place des sciences du langage dans la didactique du français en 2008 ?
}

\author{
Claudine Garcia-Debanc \\ Laboratoire Jacques Lordat - Centre interdisciplinaire des sciences du langage et de la cognition \\ cgarciad@toulouse.iufm.fr
}

\begin{abstract}
Depuis l'ouvrage fondateur de Genouvrier et Peytard (1970) Linguistique et enseignement du français, la question des relations entre sciences du langage et didactique du français s'est affinée. Linguistique appliquée ? Impliquée ? Convoquée ? Comment s'opère la circulation entre les problématiques de recherche en didactique du français et l'avancée des sciences du langage?
\end{abstract}

Si l'on prend comme concept central en didactique le concept de transposition didactique (Chevallard, 1991) comme analyse des écarts entre théories de référence et contenus à enseigner, contenus à enseigner et contenus effectivement enseignés, contenus enseignés et connaissances acquises par les élèves, on mesure toute l'importance d'une référence aux travaux de recherche linguistiques et psycholinguistiques pour analyser les matériels d'enseignement, les interactions en classe et leur effets sur les apprentissages des élèves.

L'intervention s'appliquera à montrer, à partir d'exemples de recherches didactiques portant sur les interactions orales en classe, sur la production écrite et sur l'enseignement de la langue, notamment du lexique, comment un ancrage fort de la didactique du français dans les sciences du langage garantit une exigence dans la réflexion sur le fonctionnement de la langue. Elle montrera quels concepts issus des recherches linguistiques sont utilisés mais aussi quelles sont les spécificités des recherches didactiques par rapport aux recherches linguistiques appliquées à des corpus scolaires.

Les questions qui seront posées seront plus particulièrement celles-ci. Quelle est la spécificité des questions de recherche que se posent les didacticiens ? Lorsque des recherches en didactique du français convoquent des notions empruntées aux sciences du langage pour analyser des productions d'élèves ou d'enseignants, comment s'articulent l'analyse linguistique du corpus et l'analyse didactique ? Qu'est-ce qui distingue une étude didactique d'une analyse de corpus en linguistique ?

A partir des interventions proposées dans le cadre de ce colloque mais aussi, plus largement, des travaux actuels conduits en didactique du français, l'intervention interrogera la part relative de recherches consacrées à définir des théories de référence utiles pour l'enseignement, analyser de façon critique du matériel d'enseignement, décrire et analyser les productions des élèves et des enseignants, interpréter les analyses dans un cadre didactique et valider les effets des interventions didactiques proposées. Elle questionnera les problématiques travaillées et les zones d'ombre. Les exemples présentés permettront d'interroger l'état des travaux en didactique du français mais aussi de montrer que les recherches didactiques peuvent poser en retour quelques questions aux théories linguistiques de référence. 